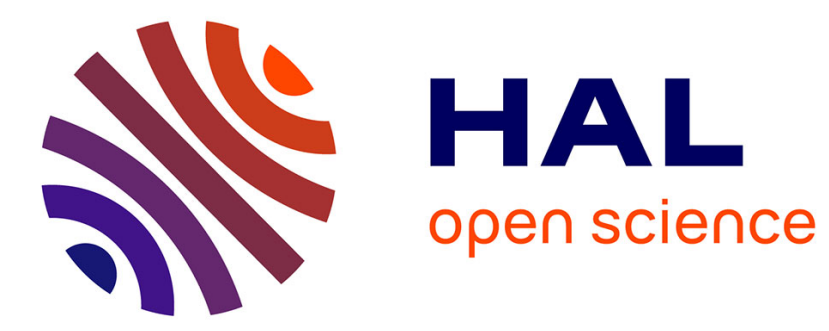

\title{
Array processing for the localisation of noise sources in hot flows
}

\author{
Ifanila Rakotoarisoa, David Marx, Christian Prax, Vincent Valeau
}

\section{To cite this version:}

Ifanila Rakotoarisoa, David Marx, Christian Prax, Vincent Valeau. Array processing for the localisation of noise sources in hot flows. Mechanical Systems and Signal Processing, 2019, 116, pp.160-172. 10.1016/j.ymssp.2018.06.038 . hal-02281494

\section{HAL Id: hal-02281494 \\ https://hal.science/hal-02281494}

Submitted on 21 Nov 2019

HAL is a multi-disciplinary open access archive for the deposit and dissemination of scientific research documents, whether they are published or not. The documents may come from teaching and research institutions in France or abroad, or from public or private research centers.
L'archive ouverte pluridisciplinaire HAL, est destinée au dépôt et à la diffusion de documents scientifiques de niveau recherche, publiés ou non, émanant des établissements d'enseignement et de recherche français ou étrangers, des laboratoires publics ou privés. 


\title{
Array processing for the localisation of noise sources in hot flows
}

\author{
Ifanila Rakotoarisoa, David Marx*, Christian Prax, Vincent Valeau \\ Institut PPRIME UPR 3346, CNRS - Université de Poitiers - ENSMA, Bât. B17 - 6 rue Marcel Doré - TSA 41105, 86073 Poitiers Cedex 9, France
}

\begin{abstract}
This paper investigates the problem of localizing a sound source in a heated flow using a microphone array. Applications are found in studies dealing with the identification of sound sources in hot turbulent jets, or with the sound radiation from installed turbofans. Two configurations have been investigated: a shear layer flow (wind-tunnel type) and a jet flow. In the present study acoustic data are generated using a simulation based on the Linearized Euler Equations. For heated flows, refraction by temperature gradients is superimposed with refraction by velocity gradients, and the objective of this study is to assess whether this effect is important and how it can be accounted for in different source localisation methods. For this purpose, a time-reversal-based imaging method has been compared with a beamforming-based method in which the time-delays are computed based on ray tracing. For the shear flow, the results show that for high subsonic Mach numbers and steep thermal gradients, the thermal stratification must be taken into account to ensure a satisfactory precision of localisation for both methods. However, including the gradients of velocity and temperature is less crucial for imaging sound sources in the jet flow. The results indicate also that the localisation error is lower with the beamforming and ray-tracing technique than with the time-reversal technique, the latter being more sensitive to the limited array aperture.
\end{abstract}

\section{Introduction}

In the context of aeroacoustic measurements in laboratories, two configurations are often encountered: (i) the case of a uniform flow separated from the medium at rest by a shear layer, which is a situation typical of open wind-tunnel flows, that will be called shear layer flow in the rest of the paper; (ii) the case of a free turbulent jet. In both cases, one classical issue is to describe the aeroacoustic sources, particularly in terms of spatial distribution and level, by using an array of microphones. The specificity of this problem is that the array of microphones used to record the noise is installed outside the flow. Consequently, before reaching each transducer, the acoustic waves undergo some convection and refraction effects induced by the flow profile. That is why ideally, for warranting reliable results, any phased-array method must include a propagation model that takes into account the spatial variations of the medium characteristics, such as the mean flow velocity [1], but also, if the flow is anisothermal, its temperature and density.

For the case of shear flows, Amiet [2] suggests a simplified analytical model to geometrically assess the refraction effects when the acoustic waves cross the the shear layer. In aeroacoustic studies, such a model is generally integrated to

\footnotetext{
* Corresponding author.

E-mail address: david.marx@univ-poitiers.fr (D. Marx).
} 
the beamforming technique [1], for calculating the propagation times in the propagation model. It has been validated both numerically [3] and experimentally [4] in the cases of bi-dimensional (aerofoil) and tri-dimensional (landing gear) shear flows. The Amiet model is limited to the simple case of an infinitely thin shear layer, and not applicable to turbulent jet flows. A more sophisticated (but more expensive computationally) approach consists in calculating the time delays required for beamforming by using a ray-tracing model [1]; the advantage of such an approach is that the ray-tracing estimation is much more flexible as it can deal with any arbitrary flow profile, although it is limited by a high-frequency approximation, ie., the acoustic wavelength is smaller than the inhomogeneities variation scales in the flows.

Acoustic sources in flows can also be located by using an imaging technique based on the time-reversal (TR) principle. Such a technique has been extensively developed since the late eighties for a medium at rest $[5,6]$, and the applicability of the technique to flows was further discussed by Roux et al. [7]. A first implementation of the TR technique to aeroacoustic applications was proposed by Deneuve et al. [8,9], based on numerical aeroacoustic data. The application of the TR principle to experimental data followed in 2012 [10]. Further developments and aeroacoustic applications were then proposed following those works [11-17]. In this paper, a localisation method based on the TR principle is used, based on the initial works of Padois et al. [10]. The radiated field is first measured with an array of microphones, either experimentally or by using simulated data. In a second step, a numerical code is used to simulate a TR process. For this purpose, the stored signals are played backward by each element of the array; the produced waves create a beam that focuses on the position of the initial source, which is commonly called back-propagation or sound focusing. Using this process, it is possible to reconstruct the acoustic field of the source and to use it for the localisation. One of the most important benefits of a numerical TR-based method for localizing sources in flows is the exact implementation of the refraction effects due to the gradients of velocity, and possibly those of temperature induced by the flow. A TR-based method is then applicable to any kind of shear flow, including jet flows.

This paper investigates how a classical source imaging technique, the beamforming technique, and one relatively more recent technique, based on the TR principle, can be used for localizing sources in heated flows. Relevant applications are found in studies dealing with the identification of sound sources in hot turbulent jets, or with the sound radiation from installed turbofans; this last case correspond to a heated shear flow. To our knowledge, no report is available on studying the possible implication of temperature effect on phased array results. Indeed, for example, sound sources in jets are typically carried out with no specific models for waves propagation through the jet flow (eg., [18]), and studies dealing with phased-array measurements in the case of hot jets do not report any attempt of taking into account temperature effects in the used propagation model [19].

Following those observations, two objectives justify the fully numerical study presented in this paper. The first one is to assess the ability of two array-processing methods: (i) the beamforming technique associated to a ray-tracing code, and (ii) the TR-based numerical method, to locate a harmonic point source in a non-homogeneous medium including gradients of velocity and temperature. The other objective is to discuss the usefulness of including the temperature gradients into the model of the medium; two distinct types of flows are considered, the shear layer flow and the jet flow. In Section 2, the numerical method used to generate the acoustic data and both array processing methods are described. In Section 3, the generated acoustic fields are investigated in terms of flow and temperature effects, in order to allow a better understanding of the array processing results, which are presented in Section 4. The cases of the shear layer flow and jet flow are analysed separately, and some conclusions are drawn about the benefits of the inclusion of temperature and velocity effects in the sound propagation model for each inverse method. Finally, some concluding remarks are provided at the end of this paper.

\section{Methodology}

\subsection{Numerical data generation}

The inverse problem considered in the following is the localisation of a harmonic point source in a flow based on the time histories of the acoustic pressure at several points, which are recorded by a linear array of sensors. In the present study these input data are generated numerically. The two-dimensional simulation of acoustic waves propagation is performed through the resolution of the Linearized Euler Equations (LEE) in the space-time domain $[20,21,10]$.

A harmonic point-like source emitting at $f_{0}=5 \mathrm{kHz}$, whose time evolution is proportional to $\sin \left(2 \pi f_{0} t\right)$, is placed at the origin of a bi-dimensional computational domain of dimensions $L_{x} \times L_{y}=1.29 \mathrm{~m} \times 2.18 \mathrm{~m}$. The linear array where the acoustic pressure is recorded has a fixed length of $L=15 \lambda_{\infty}$ and is placed at a fixed distance of $R$ from the point source (Fig. 1(a)), where $\lambda_{\infty}$ is the acoustic wavelength in the medium at rest in standard conditions of temperature and pressure. Subsequently, $R=15 \lambda_{\infty}$ for the shear layer and $R=14 \lambda_{\infty}$ for the jet. The propagation medium is animated by a parallel and stationary mean flow. In this study, we consider two different models of parallel flows: a shear layer flow (wind-tunnel-type) and a Gaussian-profile flow (jet-type). In both cases, the maximum velocity is denoted by $u_{\mathrm{f}}$ ("f" stands for "flow"). The shear layer flow is defined by a transverse profile of the velocity $u_{0}(y)$ [3]:

$$
u_{0}(y)=\frac{u_{\mathrm{f}}}{2}\left(1-\tanh \left[\frac{2\left(y-y_{s}\right)}{b_{s}}\right]\right),
$$


(a)

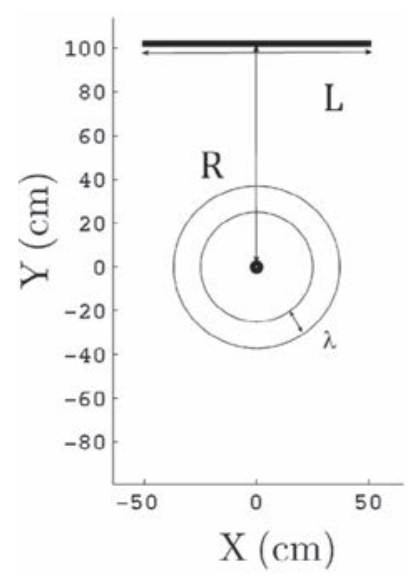

(b)

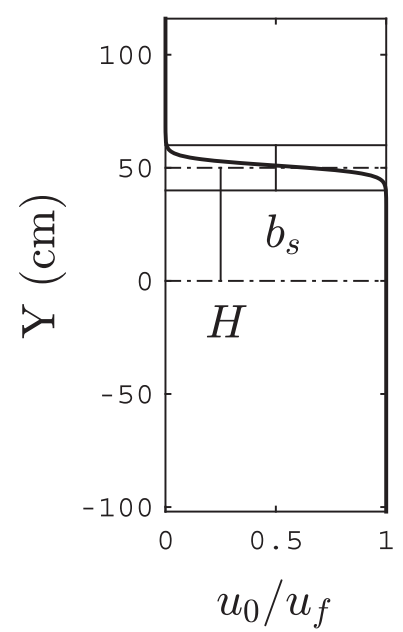

(c)

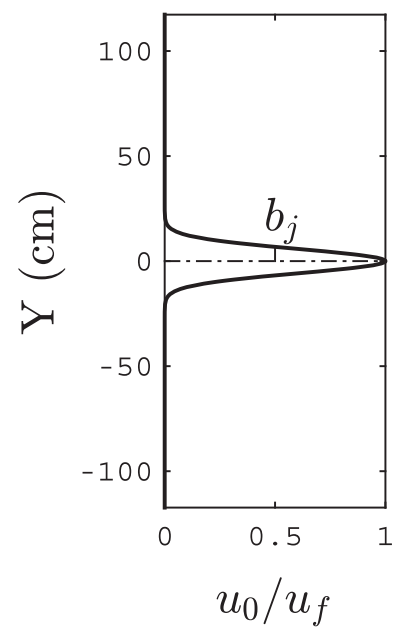

Fig. 1. (a) Array configuration relatively to the sound source position located at the origin of the computational domain. Profiles of velocity for (b) the shear layer flow, (c) the jet flow. The variable geometrical parameters of the problem are: the shear layer thickness $b_{s}$, the jet width $b_{j}$. The fixed parameters are the array-source distance $R$, the array length $L$, the acoustic wavelength in the medium at rest $\lambda_{\infty}$ and the flow height $H$ (in the shear layer flow case).

where $y_{s}$ is the position of the inflection point of the shear layer and $b_{s}$ denotes its thickness. The flow height $H$ is defined as the distance from the source location to this inflexion point. In this study, $H$ is equal to the half of the source-array distance, i.e., $H=R / 2$.

The profile of the jet is given by:

$$
u_{0}(y)=u_{\mathrm{f}} \exp \left[-\ln 2\left(\frac{y}{b_{j}}\right)^{2}\right]
$$

where $b_{j}$ is the half-width of the jet at half the maximum velocity $u_{\mathrm{f}}$. The latter is defined by the Mach number $\mathcal{M}=u_{\mathrm{f}} / c_{\infty}$, the chosen values of which are $\mathcal{M}=[0.2 ; 0.6 ; 0.9]$ in this study, $c_{\infty}$ being the speed of sound in the medium at rest with standard conditions for temperature and pressure. Fig. 1(b) and (c) display the transverse profiles of velocity for both flow configurations (wind tunnel and jet).

The maximum temperature $T_{\mathrm{f}}$ in the core of the flow will always be greater than or equal to the temperature $T_{\infty}$ of the ambient medium, in order to model the practical case of a heated flow. The effect of temperature on propagation is modelled numerically by taking into account a density profile. In shear flows, it is usual to deduce a mean density or temperature profile from the mean velocity profile by using the Crocco-Busemann relation [22]. According to this relation, in the perfect-gas hypothesis, for a Prandtl number $\operatorname{Pr}=1$, and for a uniform pressure $p_{0}$, the mean density profile $\rho_{0}(y)$ can be written as a function of the velocity profile through the expression [23]:

$$
\rho_{0}(y)=\left[-\frac{1}{2} \frac{\gamma-1}{\gamma p_{0}}\left(u_{0}(y)-u_{\mathrm{f}}\right) u_{0}(y)+\frac{1}{\rho_{\mathrm{f}}} \frac{u_{0}(y)}{u_{\mathrm{f}}}+\frac{1}{\rho_{\infty}} \frac{u_{\mathrm{f}}-u_{0}(y)}{u_{\mathrm{f}}}\right]^{-1},
$$

where $\gamma$ is the ratio of specific heats, $\rho_{\infty}$ is the density of the ambient medium, and $\rho_{\mathrm{f}}$ is the minimum density that corresponds to the maximum velocity $u_{f}$ in the shear layer or in the jet. It is also related to the maximum temperature $T_{f}$ in the flow by the perfect-gas law: $\rho_{\mathrm{f}}=\frac{p_{0}}{r_{g} T_{f}}$ where $r_{g}$ is the gas constant. The temperature profile corresponding to the density profile is $T_{0}(y)=p_{0} /\left(r_{g} \rho_{0}(y)\right)$. The temperature in the core of the flow takes values $T_{\mathrm{f}}$ so that $T_{\mathrm{f}} / T_{\infty}=[1 ; 1,5 ; 2 ; 3]$. The parameters $b_{s}$ and $b_{j}$ take the values $\left[0.1 \lambda_{\infty} ; \lambda_{\infty}\right]$. Consequently, the Strouhal number $S t=\frac{2 b_{j}}{\lambda_{\infty} \mathcal{M}}$ (for the jet) is in the range [0.22 10], which corresponds to typical frequency spectra of jet noise [24].

The numerical methods to solve the LEE are presented in [10,13]. The spatial derivatives are computed through a dispersion-relation-preserving finite-difference scheme [25] and time integration is performed with a Runge-Kutta scheme of order 4. Mesh refinement is applied to both flow configurations and ensure that the shear regions are descretized with a minimum of 10 points (per $b_{s}$ or $b_{j}$ ) in the transverse direction. It has been verified that a grid refinement by a factor of 2 does not change the results. The numerical linear array of sensors is made of adjacent grid points along the $x$-axis where acoustic fluctuations of pressure, density, and velocity are stored at each time step during the simulation time, noted T. A well-known numerical difficulty with the LEE is that the emission of acoustic waves is likely to excite the shear layer instability waves of the Kevin-Helmholtz kind. Therefore, acoustics might be overshadowed by aerodynamics. In order to focus the study on the 
refraction effects, it seems necessary to filter out these instabilities. Here we choose to simply remove the term involving the gradient of the mean flow that is the cause of the instability, as proposed by Bogey et al. [26].

\subsection{Array processing methods}

\subsubsection{Time-reversal imaging}

A first strategy to localize the source is to use time reversal, as presented in Padois et al. [10] and Rakotoarisoa et al. [13]. This consists in performing the back-propagation of the acoustic waves, from the sensors back to the source. The source positions are then identified as the points where the sound wave re-focus. During this process, the linear array, called timereversal mirror (TRM), is used in the re-emission mode. That is, the acoustic fluctuations of pressure $p_{\text {rec }}$, density $\rho_{\text {rec }}$ and velocity $\mathbf{u}_{\text {rec }}$ recorded at the array are time-reversed, or equivalently played in reverse order, according to:

$$
\begin{aligned}
& p(\mathbf{r}, t)=p_{\text {rec }}(\mathbf{r}, T-t), \\
& \rho(\mathbf{r}, t)=\rho_{\text {rec }}(\mathbf{r}, T-t), \\
& \mathbf{u}(\mathbf{r}, t)=-\mathbf{u}_{\text {rec }}(\mathbf{r}, T-t), \forall \mathbf{r} \in \Sigma,
\end{aligned}
$$

where $\Sigma$ is the area covered by the array. In addition, to ensure the full time-invariance, the mean flow must be reversed in the whole computational domain $\mathcal{D}$ :

$$
\underbrace{\mathbf{u}_{0}(\mathbf{r})}_{\text {backward propa }}-\underbrace{\mathbf{u}_{0}(\mathbf{r})}_{\text {direct propa }}, \quad \forall \mathbf{r} \in \mathcal{D} \text {. }
$$

In short, this relation says that if a wave front has been convected in one direction during the direct (forward) propagation, it needs to be convected in the opposite direction during the backward propagation for this front to return to the spot it was emitted from.

Technically, the back-propagation is simulated numerically using the LEE, with exactly the same solver that has been used to produce the data during the direct problem. Only the flow direction is reversed according to Eq. (7), while the density profile in Eq. (3) is the same as during the direct problem. From the simulated back-propagated field, the spatial distribution of the pressure root mean square (RMS) is computed in order to identify the sound source, as illustrated below in Section 4. In the rest of the paper, this method will be denoted by TRI (Time-Reversal Imaging).

\subsubsection{Beamforming with ray tracing}

The second imaging method is built from the conventional beamforming technique in the frequency domain $[1,27]$. Mathematically, for a given angular frequency $\omega$, the output of a beamformer at a given position $\mathbf{x}_{\mathbf{f}}$ can be written as follows:

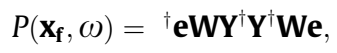

where the symbol ${ }^{\dagger}$. denotes a conjugation and transposition operator; $\mathbf{W}$ is a $M$-by- $M$ diagonal matrix ( $M$ being the number of microphones) containing the weighting coefficients affected to each transducer (if necessary) and $\mathbf{Y}^{\dagger} \mathbf{Y}$ is the $M$-by- $M$ cross-spectral matrix built from the values at frequency $\omega$ of the $M$ Fourier transforms of the recorded signals. A term crucial in Eq. (8) for the accuracy of the method is the steering vector $\mathbf{e}$. It is an M-element column vector that defines theoretically the characteristics of the propagation medium, based on the Green function of the propagation equation. The calculation of $\mathbf{e}$ is based on the accurate estimation of the time delays $\tau_{m f}$ between a focusing point located at $\mathbf{x}_{\mathbf{f}}$ in the scanning domain and each microphone $m$, according to $e_{m}=\sqrt{r_{m f} / r_{c f}} \exp \left(-\mathrm{j} 2 \pi f \tau_{m f}\right)$ [28] ( $f$ is the frequency). The distance-related amplitude is taken into account through the ratio $\sqrt{r_{m f} / r_{c f}}$ (where the square root accounts for a two-dimensional space), the microphone-scanning point distance $r_{m f}$ being normalized to the distance $r_{c f}$ from the scanning point to the array geometrical center.

However, analytical formulations of the Green function in flows are only available for uniform free flows. In the presence of boundaries (such as in closed wind tunnels), an image source model can be used to estimate the Green function [29]. In the case of free shear flows (such as in open wind tunnels), some approximations of the time delays can be obtained from the classical model of Amiet [2], but this model is not well-adapted to jet-type and/or arbitrary anisothermal flows. For this reason, in this study, we couple the conventional beamforming technique with a physical model that takes into account the spatial variations of both the velocity $u_{0}(y)$ and the density $\rho_{0}(y)$ (or, equivalently, the temperature $T_{0}(y)$ with mean pressure $p_{0}$ assumed to be uniform).

For this purpose, the calculation of the time delays is performed through the ray theory. The underlying equations will not be presented exhaustively: we invite the reader to refer, for example, to the work of Candel [30] for further theoretical details. A basic assumption of this model is that the scale of variation of the propagation medium is large compared to the sound wavelength. Following such a high-frequency approximation hypothesis, the calculation of the path followed by each ray can be carried out by numerically solving the following set of differential equations (the index notation $i$ denotes the component along each spatial dimension): 


$$
\begin{aligned}
\frac{d x_{i}}{d t} & =c \frac{k_{i}}{k}+u_{0, i} \\
\frac{d k_{i}}{d t} & =-k \frac{\partial c}{\partial x_{i}}-k_{j} \frac{\partial u_{0, j}}{\partial x_{i}},
\end{aligned}
$$

where $\mathbf{x}$ is the position along the path, $\mathbf{k}$ is the wave vector, and $c$ is the local speed of sound, value of which is computed from the mean temperature profile, $T_{0}(y)$. Eq. (9) describes the wavefront convection, while Eq. (10) defines the refraction effects induced by the gradients of density (following Eq. (3)), and velocity (according to Eqs. (1) or (2)). A numerical ray shot consists in calculating the pair $(\mathbf{x}, \mathbf{k})$ from a starting point, located in the source region to be scanned (with a starting ray angle), to an ending point by integrating Eqs. (9) and (10) using an Adams-Bashforth-Moulton predictor-corrector method (ode113 in matlab). The process is repeated for several initial starting angles. The time at which the rays eventually cross the antenna provide the time delay between the source and the microphones (some interpolation may be needed). If a source point is not connected to a particular microphone in the antenna by any ray, the corresponding weight in $\mathbf{W}$ (see Eq. (8)) is set to zero. In the following, the resulting method of localisation will be denoted by BF + Ray (BeamForming with Ray tracing).

\section{Generated acoustic fields}

To better understand the localisation results obtained from the above-mentioned localisation techniques, it is first necessary to observe how the acoustic field emitted by the source is affected by the convection and refraction effects induced by both temperature and velocity gradients. In all the results presented in this paper, the flow goes from the left to the right of the computational domain.

\subsection{Shear layer flow}

Figs. 2(a) and (d) show some pressure snapshots obtained during direct propagation with the LEE solver, together with the corresponding RMS pressure (Figs. 2(b) and (e)) and the matching ray tracing results (Figs. 2(c) and (f)) obtained at
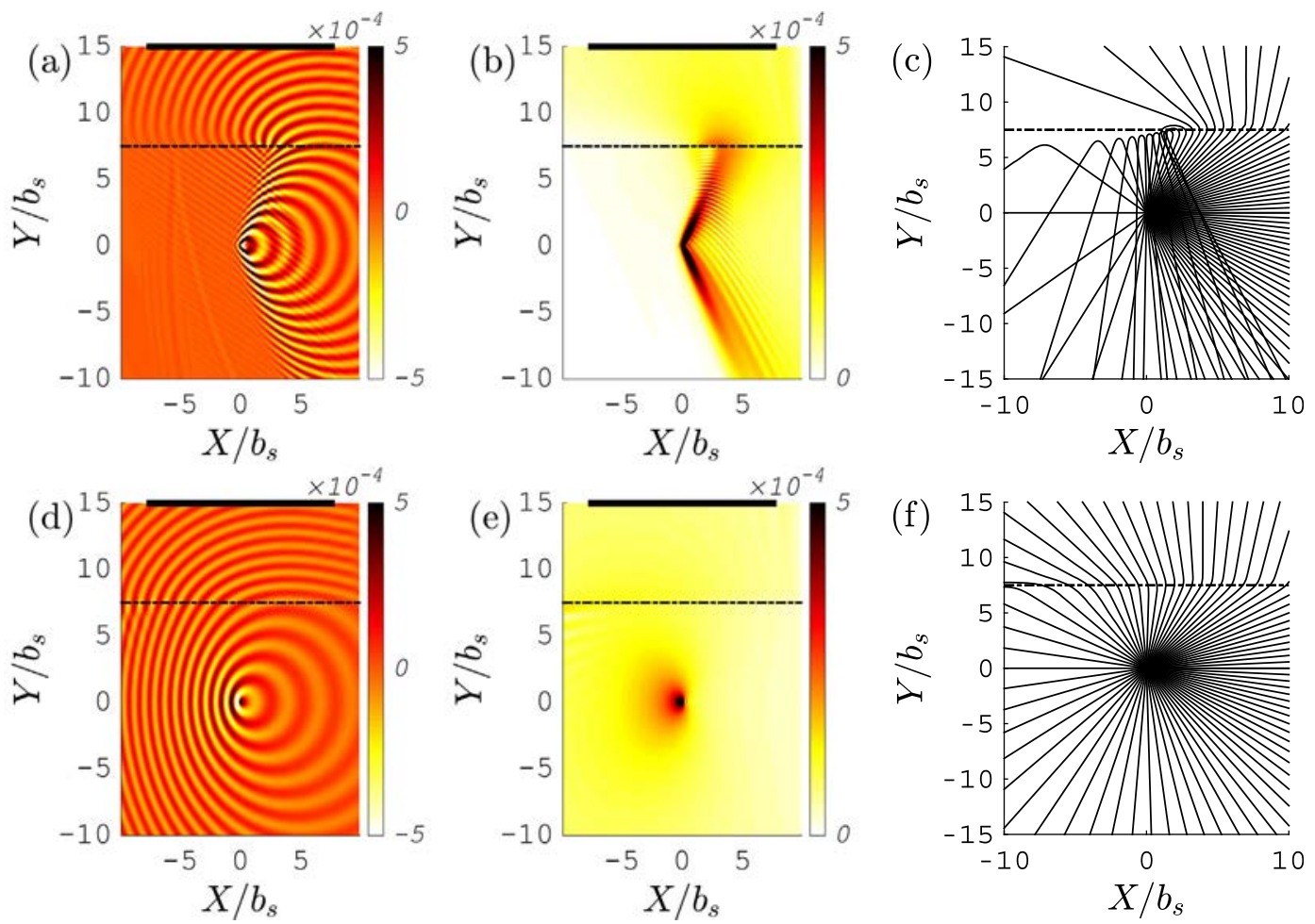

Fig. 2. Radiation of a harmonic source located at the origin of the computation domain, in a shear layer flow $\left(\mathcal{M}=0.9, b_{s} / \lambda_{\infty}=1\right)$. The inflection point of the shear layer is located at at $Y / b_{s}=7.5$ (indicated by a dash-dotted horizontal line). (a,b,c) $T_{f} / T_{\infty}=1 ;$ (d,e,f) $T_{f} / T_{\infty}=3$. Left column: particular snapshots of the acoustic pressure from numerical simulations; middle column: distribution of the RMS pressure field; right column: ray tracing. The thick horizontal lines in the color maps indicate the position of the microphone antenna. 
$\mathcal{M}=0.9$ for two different temperatures of the shear layer. It must be emphasized that those two cases are taken at the same flow speed $u_{f}$, the Mach number being defined relatively to $c_{\infty}$.

On the one hand, in the isothermal case (Fig. 2(a-b)), we note an area of relative quietness upstream the source; since the flow regime is nearly transonic, the waves practically do not propagate in the upstream direction. It is also observed from the ray-tracing calculation (Fig. 2(c)) that the waves are totally reflected by the shear layer upstream the source. On the other hand, when the flow is heated at $T_{f}=3 T_{\infty}$ (Fig. 2(d-e)), the classical pattern due to convection in flows at moderate Mach numbers is retrieved: smaller wavelength upstream (due to the decrease of the local speed of sound), and, conversely, a wavelength increase downstream. The strong dissimilarity between these acoustic fields is due to the fact that the flow temperature "compensates" for the convection effects. Indeed, the speed of sound in the hot gas is much higher than in the gas at normal temperature; in our case, their ratio is of the order of 1.7 when the gas is at rest, so that the convection effect is significantly attenuated in the hot gas. By observing the ray tracings (Fig. 2(f)), we note that the strong reflection phenomenon due to the shear layer is also nearly cancelled by the temperature.

\subsection{Jet flow}

In this second flow configuration, the shape of the wavefronts is different: they take an oval form on both sides of the jet axis as this can be observed on the snapshots in Fig. 3(a)-(d). Upstream and without any temperature gradient (Fig. 3(a)), in an area defined by $x<0$ and $|y| \leqslant 1.5 b_{j}$, the acoustic field presents an axisymmetric interference pattern. As pointed out by Candel [31], the existence of such stationary waves is due to the interaction between the waves directly emitted by the source and those reflected upstream by the flow. This phenomenon is reduced when the latter is heated, as can be observed on the snapshot in Fig. 3(d). However, in both cases a significant part of the RMS pressure shown in Fig. 3(b)(e) is located upstream of the source, and this is not intercepted by an antenna located above the flow. Another important feature of the acoustic radiation in this configuration is the cone of silence which develops downstream around the jet axis, as a consequence of the refraction effects. In particular, the "aperture" of this cone increases according to the jet temperature, refraction by the density gradients adding up to refraction by the velocity gradients.

Finally, in either wind tunnel type or jet type configurations, temperature plays an important role in the convection/ refraction process since it may practically counterbalance or increase the effect of the flow velocity.
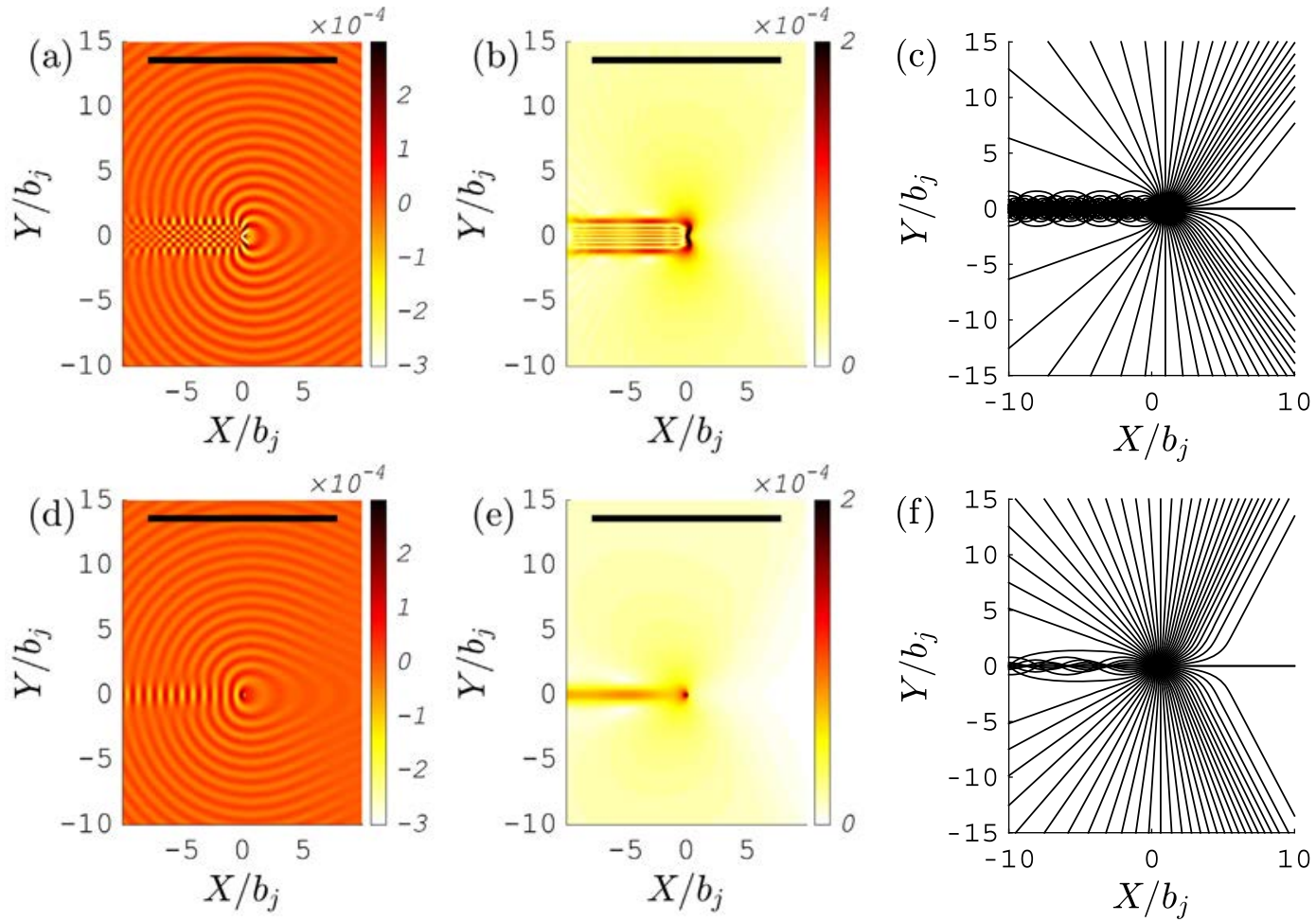

Fig. 3. Radiation of the harmonic source (located at the origin of the computation domain) in a jet flow $\left(\mathcal{M}=0.9, b_{j} / \lambda_{\infty}=1\right)$ : $(\mathrm{a}, \mathrm{b}, \mathrm{c}) T_{f} / T_{\infty}=1 ;(\mathrm{d}, \mathrm{e}, \mathrm{f})$ $T_{f} / T_{\infty}=3$. Left column: snapshots of the acoustic pressure from numerical simulations; middle column: distribution of the RMS pressure field; right column: ray tracing. The thick horizontal lines in the color maps indicate the position of the microphone antenna. 


\section{Localisation results and discussion}

\subsection{Localisation error}

This section is dedicated to the comparison of TRI and BF + Ray in the two flow configurations described previously. The observed variable is the localisation error defined as the distance separating the detected position and the actual source position. In the case of TRI, the detected position corresponds to the peak of the distribution of the RMS pressure calculated from the back-propagated acoustic field. An example is proposed for the case of the shear layer at Mach Number 0.9: in Fig. 4(a), a pressure snapshot of the back-propagated field indicates that a bended beam of periodic waves propagates from the array towards the source, undergoing the same refraction and convection effects as the waves that propagated from the source to the array [10]. In Fig. 4(b), the distribution of the RMS back-propagated field displays an energetic beam passing through the source, the maximum of which is used for estimating the position of the sound source.

In the case of the BF + Ray technique, the peak observed in Fig. 4(c) is that of the beamformer output $P$ defined in Eq. (8). To keep a coherence according to the temperature of the flow, we normalize the localisation error with the "effective" wavelength $\lambda_{\mathrm{e}}=\lambda_{\infty} \sqrt{T_{\mathrm{f}} / T_{\infty}}$. The resulting variable will be denoted as $\epsilon$.

\subsection{Shear layer flow}

In this section, the influence of several physical parameters on the normalized localisation error are investigated: the flow temperature, through the ratio $T_{\mathrm{f}} / T_{\infty}$, ranging from 1 (isothermal flow) to 3 ; the Mach number, taking the values $\mathcal{M}=[0.2 ; 0.6 ; 0.9]$; the ratio $b_{s} / \lambda_{\infty}$, taking the values $[0.1 ; 1]$, describing the thickness of the shear layer relatively to the sound wavelength. The results are presented in Fig. 5. Let us investigate the results of the TRI method. In most cases, the localisation error is relatively small and of the order of the wavelength $\lambda_{\mathrm{e}}$ independently of the velocity and the temperature. One exception is at $\mathcal{M}=0.9$ and $T_{\mathrm{f}} / T_{\infty}=1$ (isothermal medium) where the localisation error can reach nearly four wavelengths. Examples of snapshots for the direct and back-propagated pressure fields for this transonic case can be observed in Figs. 2(a) and 4(a), respectively. As already pointed out, as the source emits in nearly transonic conditions, its directivity is characterized by a downstream radiation (Fig. 2(a)). Therefore, the imaging quality is strongly affected by a lack of information on the downstream part of the array, as shown by the snapshot in Fig. 4(a) of the back-propagated pressure field. In this quite extreme case, the energetic beam is very oblique (Fig. 4(b)), and the maximum is relatively far from the source position. However, a cross-section in the $Y=0$ plane would reveal the source at its correct position; the observed error is mainly due to the poor resolution in the direction perpendicular to the array, which should be expected for a planar array. Another observation is that the localisation error is smaller for hot flows; indeed, as pointed out in Section 3 (Fig. 2), the temperature gradient attenuates the cumulated effects of convection and refraction, so that some better localisation is achieved with the time-reversal method.

Concerning the BF + Ray method the localisation errors are better than that produced by the TRI method. This is particularly true for the isothermal case $T_{f} / T_{\infty}=1$ for which the convection effects are not alleviated by temperature as it is the case at $T_{f} / T_{\infty}=3$ (see discussion of Fig. 2(a-c) vs (d-f)). This smaller error obtained by beamforming results from TRI being more sensitive to limited aperture than beamforming (see Section 4.5). It should be again noted that the difference in errors between TRI and BF + Ray is mainly obtained in the direction perpendicular to the array. If one assumes that the source lies on the line $Y=0$ parallel to the array, then both methods perform equivalently. Another interesting point is that the error obtained by beamforming is about the same for $b_{s} / \lambda_{\infty}=0.1$ (Fig. 5)) and $b_{s} / \lambda_{\infty}=1$ (Fig. 5(b)) (this is also true for TRI). This
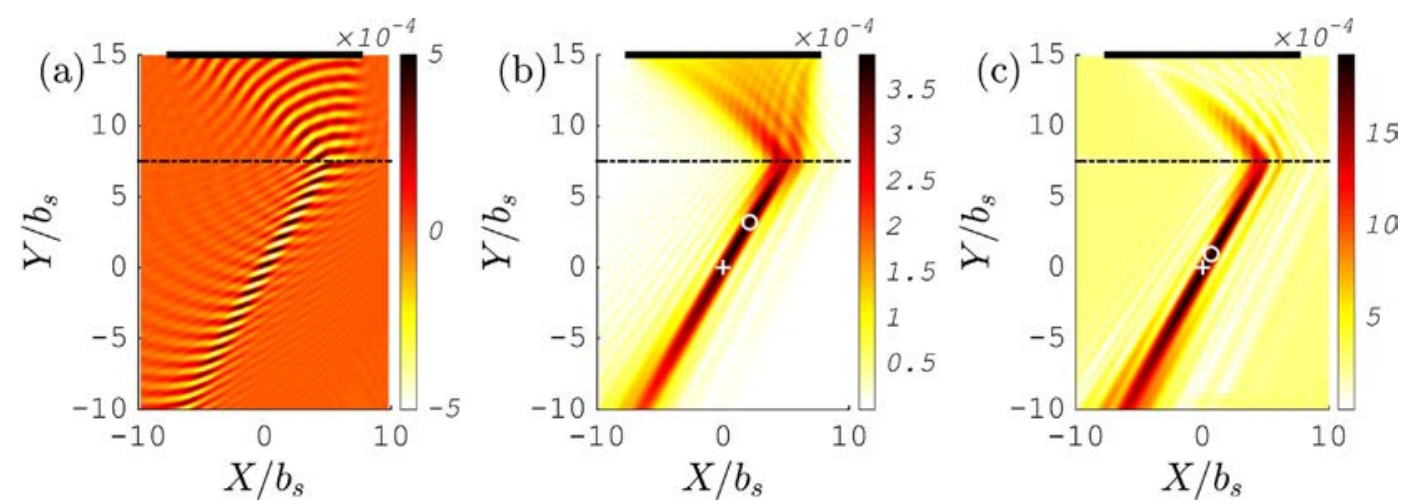

Fig. 4. Source localisation in a shear layer flow for $\mathcal{M}=0.9, T_{f} / T_{\infty}=1, b_{s} / \lambda_{\infty}=1$. The array (thick line) is located at $Y / b_{s}=15$, the inflexion point of the shear layer at $Y / b_{s}=7.5$ (dash-dotted line), and the sound source is located at the origin of the domain. (a)-(b): TR imaging with (a) Snapshot of the backpropagated pressure; (b) spatial distribution of the RMS back-propagated pressure. (c) Map obtained with BF + Ray. The cross indicates the real source position, and the circle its estimated position. The localisation error is defined as the distance between these two points. 

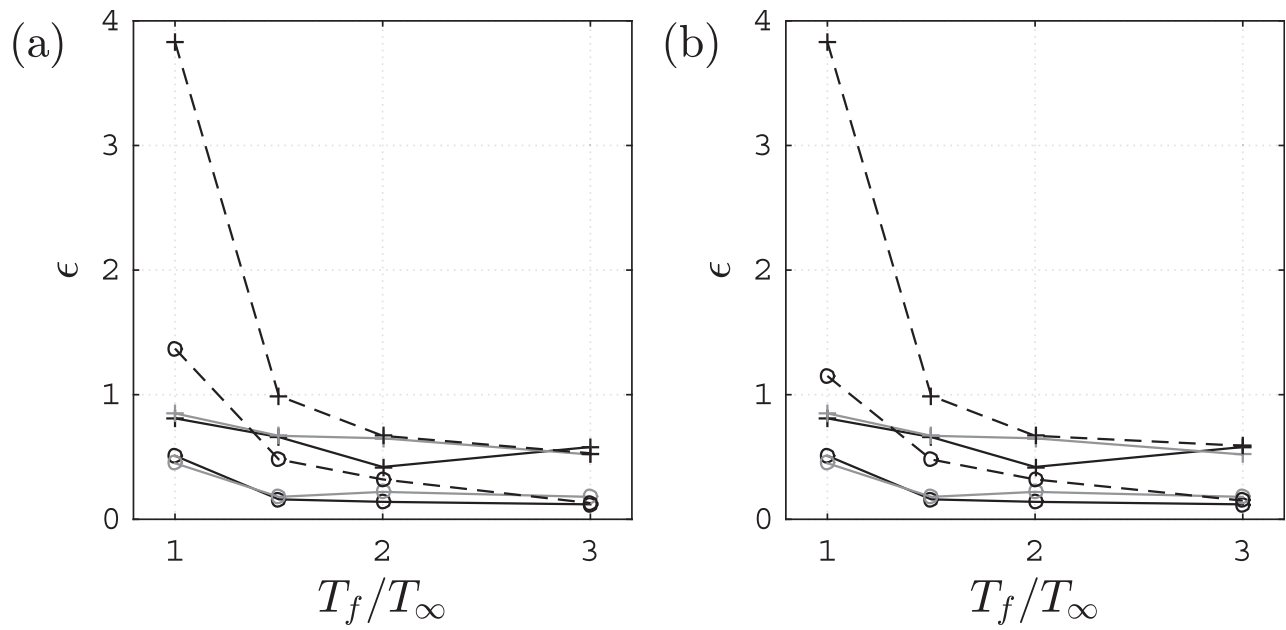

Fig. 5. Normalized localisation error for a shear layer flow (profile given by Eq. (1)) as a function of $T_{\mathrm{f}} / T_{\infty}$, obtained by TRI ( + ) and BF + Ray (o). (a) $b_{s} / \lambda_{\infty}=0.1$, and (b) $b_{s} / \lambda_{\infty}=1 . \mathcal{M}=0.2$ (black solid line), $\mathcal{M}=0.6$ (grey solid line) and $\mathcal{M}=0.9$ (black dashed line).

means that the high frequency approximation underpinning ray tracing is still statisfied even when the wavelength is much larger than the shear layer width.

\subsection{Jet flow}

An Example of source localisation for a jet flow for TRI and BF + Ray are given in Fig. 6(a-c). The energy beam exhibits a characteristic bent shape due to the cumulative effects of refraction of the two sides of the jet. However the energy beam passes through the source position, so that the localisation error shown in Fig. 7 is uniformly less than a wavelength, independently of the temperature ratio.

The fact that the waves curvature is weakly affected by the flow explains the smaller error for the jet flow case, in comparison with the shear layer case: the convection and refraction effects do not degrade significantly the information received by the array, even at transonic velocities. As for the shear layer, BF + Ray performs slightly better compared to TRI. For BF + Ray there is no much difference between the errors obtained at low frequency $b_{j} / \lambda_{\infty}=0.1$ and high frequency $b_{j} / \lambda_{\infty}=1$, which shows that the approximations made for ray tracing are not too restrictive. It is noticeable that in the lowfrequency case $\left(b_{j} / \lambda_{\infty}=0.1\right.$, Fig. $\left.7(\mathrm{a})\right)$, TRI and BF + Ray methods display consistently an error of less than half a wavelength (except at $\mathcal{M}=0.9$ ), whatever the temperature condition. However, this error is of the order of the jet diameter, which is practically a significant localisation error for making physical interpretations; this emphasizes the difficulty of applying array processing methods to jet noise sources, where the flow has characteristic dimensions typically smaller than the wavelength. The same conclusion can be drawn for TRI in the high-frequency case $\left(b_{j} / \lambda_{\infty}=1\right.$, Fig. $\left.7(\mathrm{~b})\right)$, where the localisation error is found to be around the wavelength, that is, similarly to the low-frequency case, an uncertainty equal to the jet diameter. The reason for this is explained ins Section 4.5 .
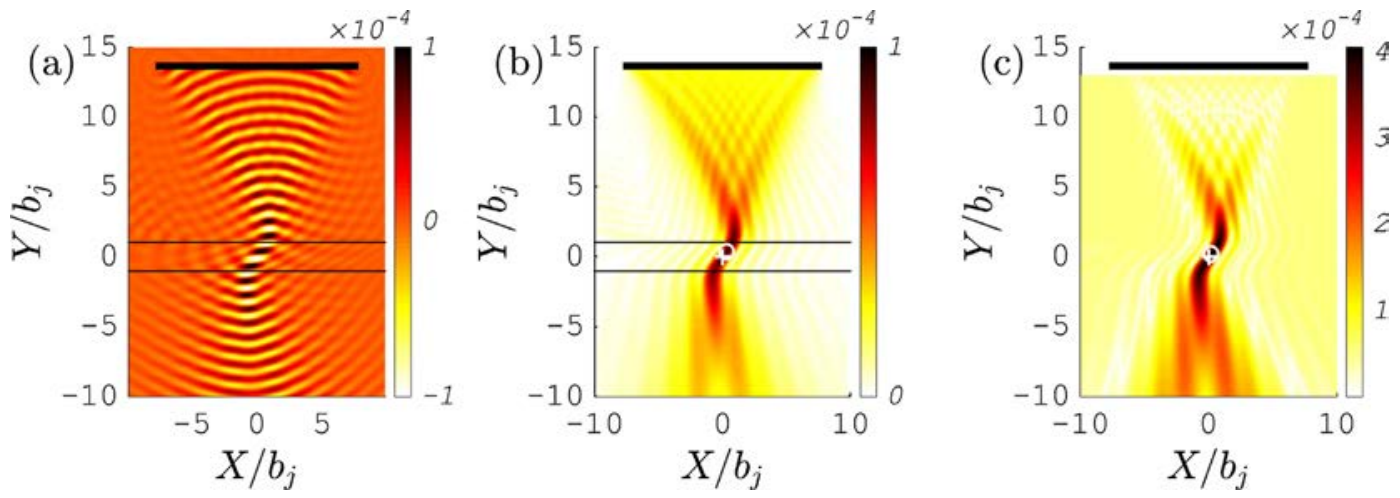

Fig. 6. Source imaging in a jet flow for $\mathcal{M}=0.9, T_{f} / T_{\infty}=1, b_{j} / \lambda_{\infty}=1$ : (a) Snapshot of the back-propagated pressure (TRI); (b) spatial distribution of the RMS back-propagated pressure (TRI); (c) BF + Ray map. The cross indicates the real source position, and the circle its estimated position. The localisation error is defined as the distance between these two points. 

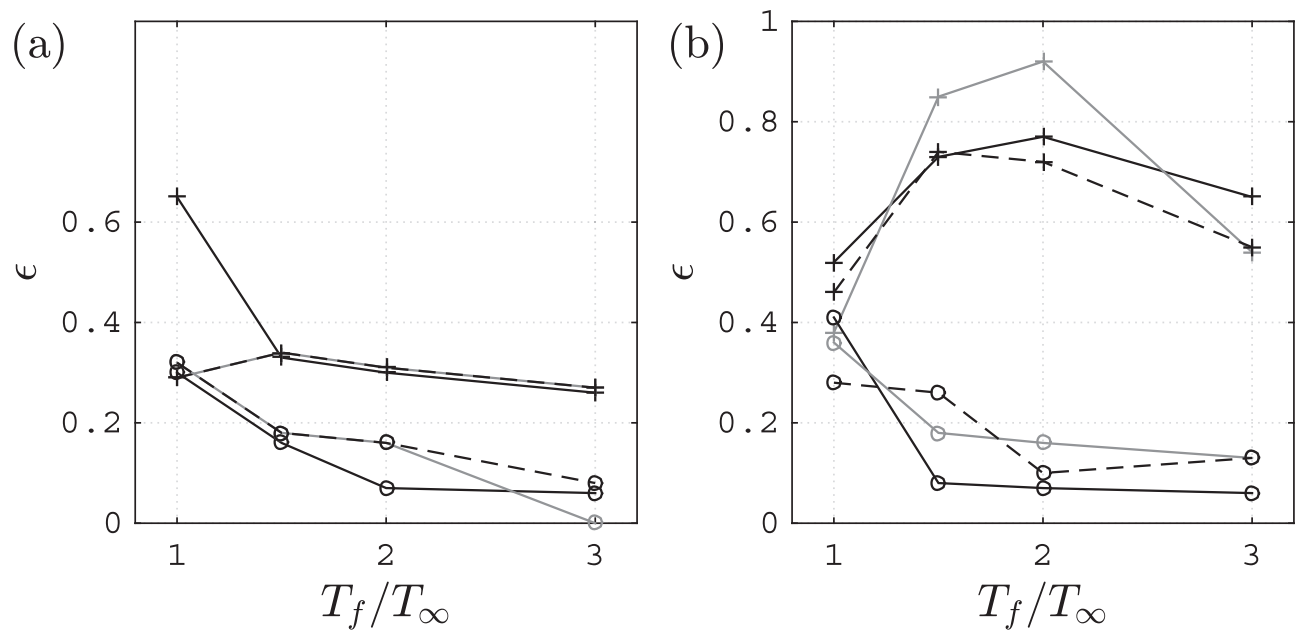

Fig. 7. Normalized localisation error for a jet flow (profile given by Eq. (2)) as a function of $T_{\mathrm{f}} / T_{\infty}$, obtained by TRI (+) and BF + Ray (o). (a) $b_{j} / \lambda_{\infty}=0.1$ and (b) $b_{j} / \lambda_{\infty}=1 . \mathcal{M}=0.2$ (black solid line), $\mathcal{M}=0.6$ (grey solid line) and $\mathcal{M}=0.9$ (black dashed line).

\subsection{Benefits of a propagation model including flow velocity and temperature gradients}

\subsubsection{Shear layer flow}

In the shear layer configuration, Fig. 8 compares, for both imaging methods, the localisation errors obtained at $\mathcal{M}=0.9$ and $b_{\mathrm{j}} / \lambda_{\infty}=1$ by taking into account or omitting the temperature and/or the velocity of the flow in the propagation model. Regarding the results obtained through TRI, we observe that the localisation error gets worse according to the flow temperature when only the velocity is taken into account. This can be explained by the observations made in the previous section: the temperature effects tend to counterbalance the velocity effects. Consequently, its omission during the focusing process amplifies the convection effects which results in a larger localisation error. The same conclusions generally hold for the BF + Ray technique, but the difference is that the error $\epsilon$ is decreasing with the flow temperature, whatever the nature of the correction in terms of velocity and temperature.

The necessity of taking into account the complete flow characteristics is emphasized by considering the results in Fig. 9, which displays the spatial distribution of the root mean square of the back-propagated field by using the TRI technique for $T_{f} / T_{\infty}=3$ and a high Mach number $\mathcal{M}=0.9$. If a quiescent propagation medium at normal temperature is considered, the source is detected clearly upstream its real position (Fig. 9(a)). If only the flow velocity profile is considered, the method is completely out of focus (Fig. 9(b)), while taking into account the complete flow characteristics (velocity and temperature) positions the energetic beam at its right location(Fig. 9(c)).
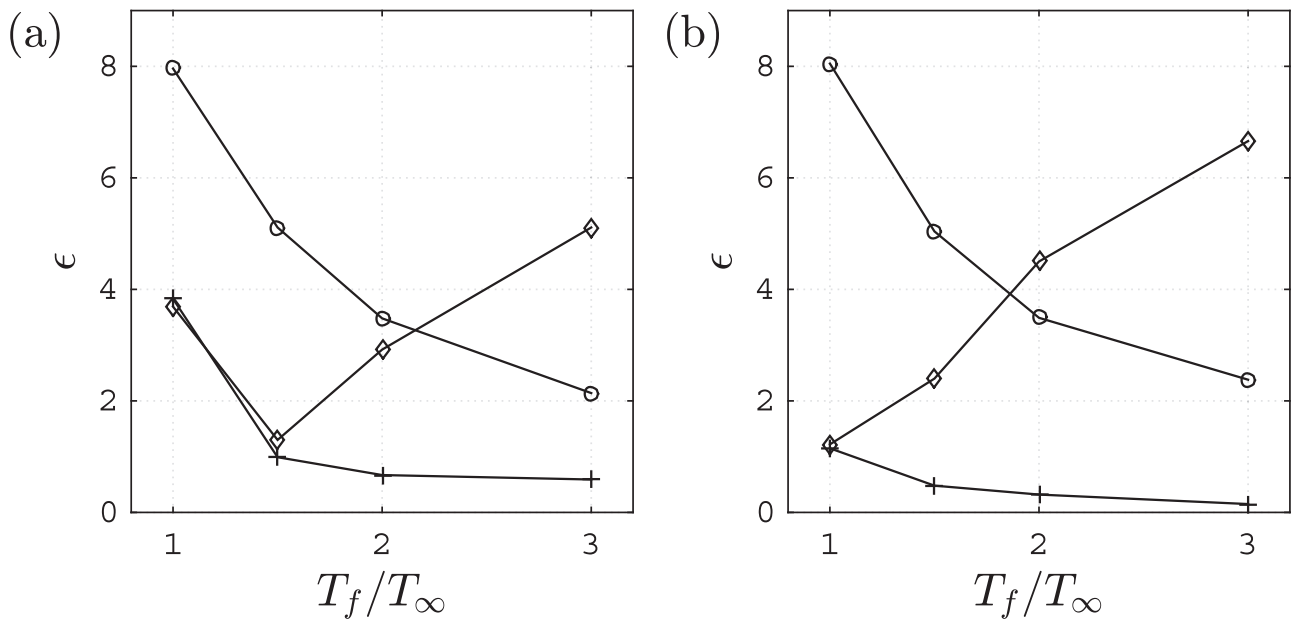

Fig. 8. Normalized localisation error in a shear layer flow $\left(\mathcal{M}=0.9, b_{s} / \lambda_{\infty}=1\right)$ by taking into account: (o) none of the flow characteristics (homogeneous medium at rest), $(\diamond)$ only the flow velocity, (+) the flow velocity and temperature. (a) TRI; (b) BF + Ray. 

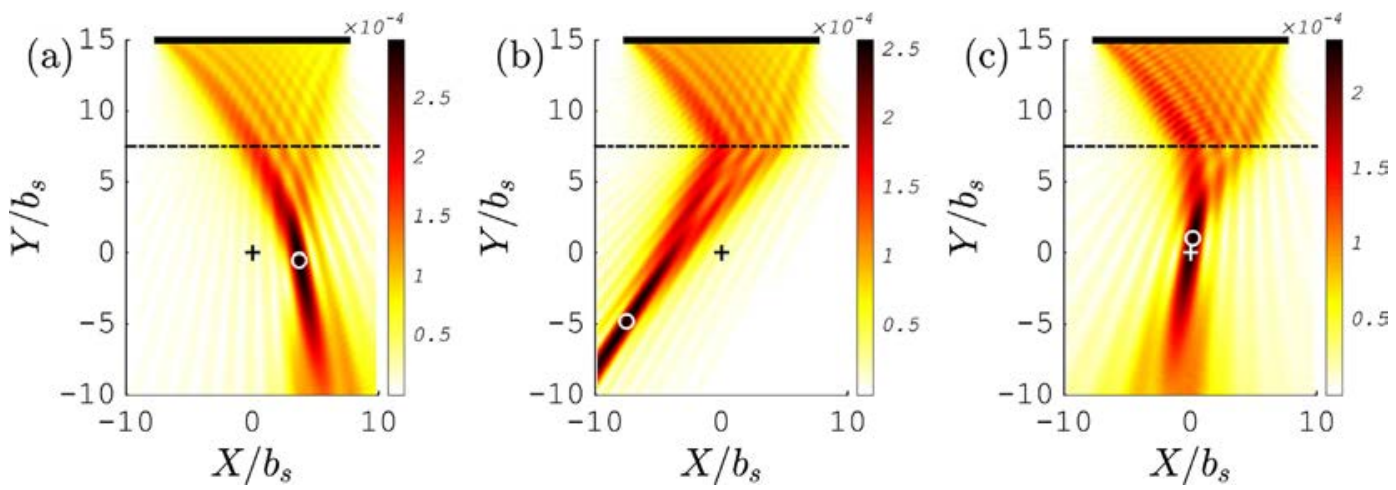

Fig. 9. TR imaging in a shear layer flow $\left(\mathcal{M}=0.9, T_{f} / T_{\infty}=3, b_{s} / \lambda_{\infty}=1\right)$, spatial distribution of the RMS the back-propagated pressure, by taking into account: (a) none of the flow characteristics (homogeneous medium at rest), (b) only the flow velocity, (c) the flow velocity and temperature. The cross indicates the real source position, and the circle its estimated position.

All these observations show that in the case of a shear flow, the knowledge of the profiles of velocity and temperature is crucial to obtain a satisfactory estimation of the source location, be it through TRI or BF + Ray. Although only the case $b_{s} / \lambda_{\infty}=1$ has been shown in this section, the conclusions remain the same for $b_{s} / \lambda_{\infty}=0.1$.

\subsubsection{Jet flow}

The same kind of results are presented in Fig. 10 in the case of a jet flow at $\mathcal{M}=0.9$; in this case, the ratio $b_{j} / \lambda_{\infty}$ is equal to 1 , so that the jet width is equal to two acoustic wavelengths. Be it through TRI or BF + Ray, the localisation error remains below the effective wavelength. Accounting or flow velocity and temperature variations leads to some improvements in the localisation for BF + Ray, and not so obvious ones for TRI. In any case, the improvement for the jet flow is less important than for the shear layer, and the same conclusion is found to hold for $b_{j} / \lambda_{\infty}=0.1$. It means that it is less crucial to take into account the gradients of velocity or temperature in the case of the jet profile. Some authors who have performed beamforming along the jet centerline have discarded the use of an advanced model for refraction [18,32], in agreement with the present results.

\subsection{Influence of array geometry on Time-Reversal Imaging}

In Figs. 5, 7, 8 and 10 it has been observed that in some cases the TRI leads to localisation errors larger than with the BF + Ray method. It turns out that the high-frequency assumption for ray tracing is not restrictive even at low Strouhal numbers such that $b_{j} / \lambda_{\infty}=0.1$, which is a necessary reason for the observed good performance of BF + Ray. However, a reason for superior preformance of $\mathrm{BF}$ is probably that in its formulation it is less dependent on the array aperture compared to time-reversal. It is indeed known that TR in a lossless medium is an inverse filter when the antenna completely surrounds
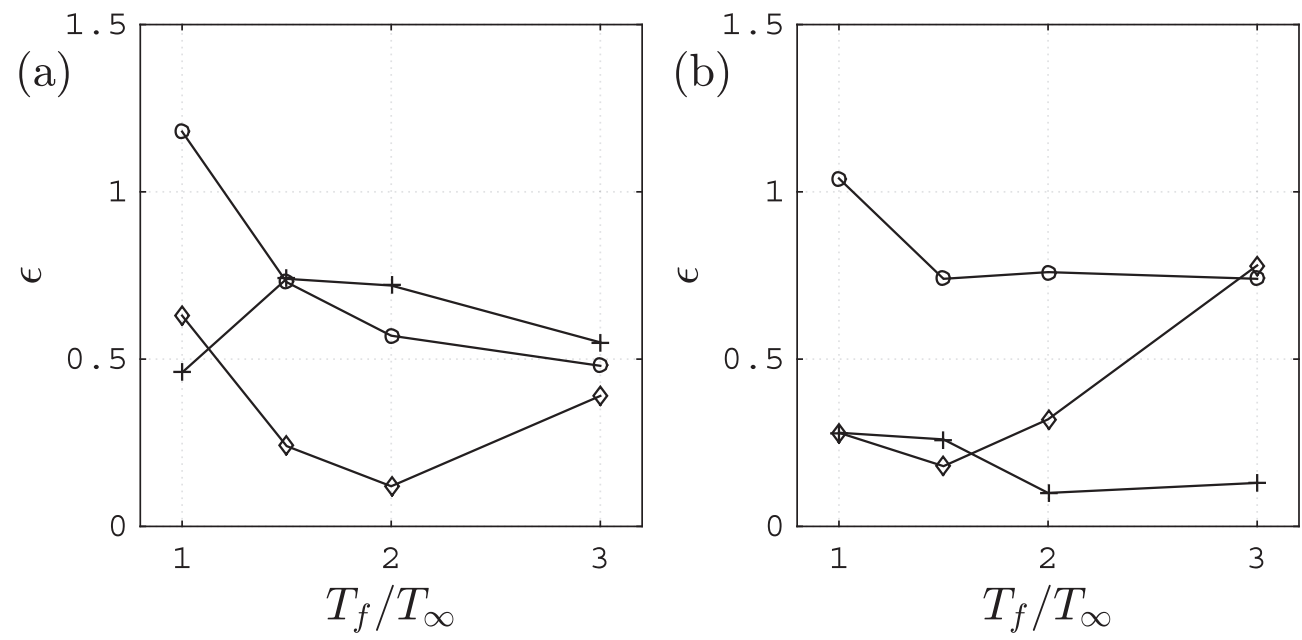

Fig. 10. Normalized localisation error in a jet flow $\left(\mathcal{M}=0.9, b_{j} / \lambda_{\infty}=1\right)$ by taking into account: (o) none of the flow characteristics (homogeneous medium at rest), $(\diamond)$ only the flow velocity, (+) the flow velocity and temperature. (a) TRI technique; (b) BF + Ray technique. 
the source [33]. Otherwise, RT is only an approximate inverse filter [33], performance of which depends on the aperture. This is investigated in the present section in Fig. 11.

The case of a heated transonic jet at Mach number 0.9 is considered $\left(T_{f} / T_{\infty}=1.5\right)$, for which the jet width is small compared to the sound wavelength (low frequency case, $b_{j} / \lambda_{\infty}=0.1$ ). If the array is located above the jet (at the same position considered until now, see Fig. 3(a) Fig. 6(a) for example), the waves back-propagating from the array are significantly reflected by the jet, due to the strong mean velocity gradient. This results in the stationary wave pattern above the jet seen in the RMS pressure map in Fig. 11(a). A section of this map along the $Y$-axis is given in Fig. 11(d). The back-propagated wave is also partly transmitted through the jet, so that a beam of energy is observed passing through the source position. However, the maximum of the RMS pressure is identified at the first node of the stationary wave pattern above the jet, and the localisation error is about 0.34 wavelength. This is larger than the error 0.18 wavelength obtained with $\mathrm{BF}+$ Ray (compare the two back dashed lines in Fig. 7(a) at $T_{f} / T_{\infty}=1.5$ ). This is also large compared to the jet dimension (at $Y=b_{j}=0.1 \lambda$ the jet velocity is decreased by a factor of 2 , at $Y=4 b_{j}=0.4 \lambda$ the jet velocity has completely vanished). In this case, the error in the axial direction of the jet is negligible, but is is clear that it is not possible to localize the source of sound in the radial direction.

If we now consider an array made of two parallel linear arrays from either side of the jet (the linear array considered in Fig. 3 (a) plus its image with respect to the $X$-axis), we observe, quite logically, that the stationary wave pattern is located on either side of the jet (Fig. 11(b) and (d)). The radial localisation is not improved compared to the case with one linear array, and the maximum of the RMS pressure field is once again located on a node of the stationary wave pattern (although there is some local but not global maximum at the source position). On the contrary, a half-cavity located in the upper half of the calculation domain (made of the linear array in Fig. 3(a) plus two vertical segments joining the extremities of this linear array to the $X$-axis) allows a perfect localisation (to mesh size accuracy), both in the axial and radial directions (Fig. 11(c) and (d)). It is then concluded that the pressure field radiated both upstream and downstream is very important for TRI, if a good performance in the radial and axial directions is required. However, such an array with a large aperture can be practically difficult to implement, in particular because the vertical part of the array located downstream should be highly exposed to the jet.
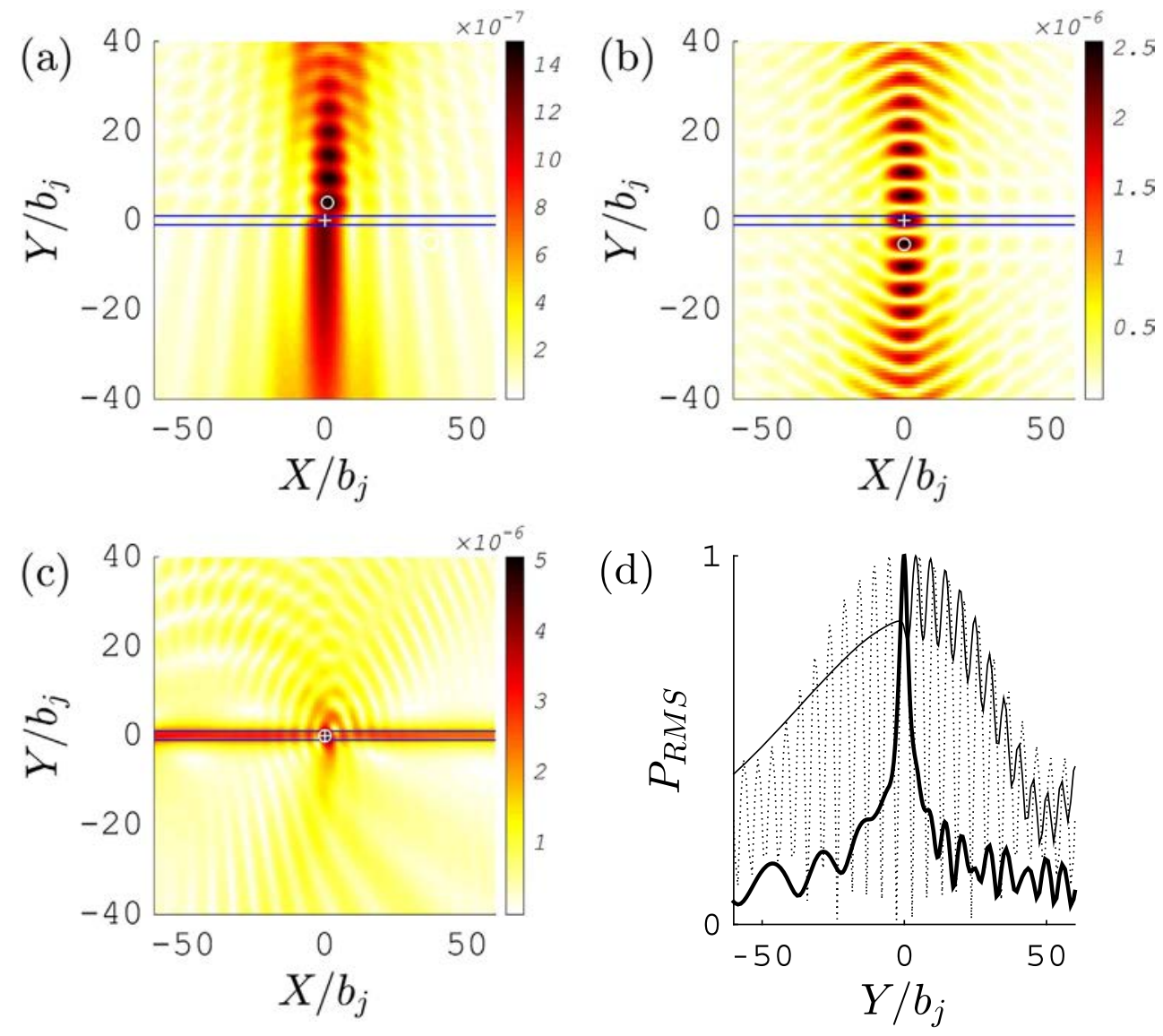

Fig. 11. Backpropagated RMS pressure obtained by TRI for a jet flow $\left(\mathcal{M}=0.9, b_{j} / \lambda_{\infty}=0.1, T_{f} / T_{\infty}=1.5\right.$ ) with different antennas: (a) top mirror; (b) top and bottom mirrors; (c) upper half-cavity. In (d) comparison of the Y-cut of RMS pressure obtained with the three different antennas (plain thin line for top mirror; dotted line for top and bottom mirrors; plain thick line for upper half-cavity). The horizontal lines in (a)-(c) indicate the limits of the core of the jet $\left(Y= \pm b_{j}\right)$, the cross indicates the real source position, and the circle its estimated position. 


\section{Conclusion}

As convection and refraction affect the propagation of sound waves from the source to the receiver, they need to be taken into account for the inverse problem of source localisation. For a heated flow, refraction by temperature gradients is superimposed to refraction by velocity gradients. The objective of this study was to assess whether this effect is important and how it can be accounted for in different source localisation methods.

A time-reversal-based imaging method has been compared to a beamforming-based method in which the computation of time-delays relies on ray tracing. Both methods take into account velocity and temperature gradients. Two configurations have been simulated: a shear layer flow (wind-tunnel type) and a jet flow, with a temperature larger than that of the ambient medium.

For the shear layer, the results show that for high subsonic Mach numbers and steep thermal gradients, taking into account thermal stratification improve the precision of the localisation, be it by TRI or BF + Ray. Two limitations have to be taken into consideration. First, the array has a limited spatial aperture and refraction by the shear layer may steer the sound wave in a direction not intercepted by the array. The time-reversal method is more sensitive to this than the beamforming method. Second, the BF + Ray imaging is based on ray tracing, which is an asymptotic method valid in the high frequency limit. Even at the lowest Strouhal number considered here (which corresponds to a flow thickness of about one tenth of the wavelength), this assumption has been shown not to be restrictive. Results also show that including the gradients of velocity and temperature is useful but less crucial for the jet flow than for the shear flow, be it for TRI or $\mathrm{BF}+$ Ray.

\section{Acknowledgement}

We thank Robin Sebastian who helped us to produce some of the beamforming results.

\section{References}

[1] T.J. Mueller, Aeroacoustic Measurements, Springer-Verlag, 2002, chapter 2.

[2] R.K. Amiet, Refraction of sound by a shear layer, J. Sound Vib. 58 (1978) 467-482.

[3] T. Padois, C. Prax, V. Valeau, Numerical validation of shear flow corrections for beamforming acoustic source localisation in open wind-tunnels, Appl. Acoust. 74 (2013) 591-601.

[4] C. Bahr, N.S. Zawodny, T. Yardibi, F. Liu, D. Wetzel, B. Bertolucci, L. Cattafesta, Shear layer time-delay correction using a non-intrusive acoustic point source, Int. J. Aeroacoustics 10 (2011) 497-530.

[5] M. Fink, C. Prada, F. Wu, D. Cassereau, Self focusing in inhomogeneous media with time reversal acoustic mirrors, in: Proceedings of the IEEE Ultrasonics Symposium, Montreal, 1989.

[6] M. Fink, Time-reversal of ultrasonic fields - Part I: basic principles, IEEE Trans. Ultrason. Ferroelec. Freq. Control 39 (1992) 555-566.

[7] P. Roux, M. Fink, Experimental evidence in acoustics of the violiation of time-reversal invariance induced by vorticity, Europhys. Lett. 32 (1995) $25-29$.

[8] A. Deneuve, P. Druault, R. Marchiano, P. Sagaut, A coupled time-reversal/complex differentiation method for aeroacoustic sensitivity analysis: towards a source detection procedure, J. Fluid Mech. 642 (2010) 181-212.

[9] E. Vergnault, O. Malaspinas, P. Sagaut, A time-reversal lattice Boltzmann method, J. Comput. Phys. 230 (2011) 8155-8167.

[10] T. Padois, C. Prax, V. Valeau, D. Marx, Experimental localization of an acoustic sound source in a wind-tunnel flow by using a numerical time-reversal technique, J. Acoust. Soc. Am. 132 (2012) 2397-2407.

[11] P. Druault, R. Marchiano, P. Sagaut, Localization of aeroacoustic sound sources in viscous flows by a time reversal method, J. Sound Vib. 332 (15) (2013) 3655-3669.

[12] A. Mimani, C.J. Doolan, P.R. Medwell, Enhancing the focal-resolution of aeroacoustic time-reversal using a point sponge-layer damping technique, JASA Express Lett. $136(3)$.

[13] I. Rakotoarisoa, J. Fischer, V. Valeau, D. Marx, C. Prax, L.-E. Brizzi, Time-domain delay-and-sum beamforming for time-reversal detection of intermittent acoustic sources in flows, J. Acoust. Soc. Am. 136 (2014) 2675-2686.

[14] A. Mimani, Z. Prime, C. Doolan, P. Medwell, A sponge-layer damping technique for aeroacoustic time-reversal, J. Sound Vib. 342 (2015) $124-151$.

[15] A. Mimani, Z. Prime, D. Moreau, C. Doolan, An experimental application of aeroacoustic time-reversal to the aeolian tone, J. Acoust. Soc. Am. 139 (2) (2016) $740-763$.

[16] A. Mimani, D. Moreau, Z. Prime, C. Doolan, Enhanced focal-resolution of dipole sources using aeroacoustic time-reversal in a wind tunnel, Mech. Syst. Signal Process. 72-73 (2016) 925-937.

[17] J. Fischer, V. Valeau, L.-E. Brizzi, Beamforming of aeroacoustic sources in the time domain: An investigation of the intermittency of the noise radiated by a forward-facing step, J. Sound Vib. 383 (2016) 464-485.

[18] S. Venkatesh, D. Polak, S. Narayanan, Beamforming algorithm for distributed source localization and its application to jet noise, AIAA J. 41 (7) (2003) $1238-1246$.

[19] S. Lee, J. Bridges, Phased-array measurements of single flow hot jets, in: AIAA/CEAS Aeroacoustics Conference, Monterey, California, 2005.

[20] C. Bailly, D. Juvé, Numerical solution of acoustic propagation problems using linearized Euler equations, AIAA J. 38 (2000) 22-29.

[21] C. Prax, F. Golansky, L. Nadal, Control of the vorticity mode in the linearized Euler equations for hybrid aeroacoustic prediction, J. Comp. Phys. 227 (2008) 6044-6057.

[22] F.W. White, second ed., Viscous fluid flow (Chapters 3 and 7), Mc Graw-Hill, 1991.

[23] A. Agarwal, P.J. Morris, Calculation of sound propagation in non-uniform flows: suppression of instability waves, AIAA J. 42 (2004) $80-88$.

[24] K. Viswanathan, Aeroacoustics of hot jets, J. Fluid Mech. 516 (2004) 39-82.

[25] C.K.W. Tam, J.C. Webb, Dispersion-relation-preserving finite difference schemes for computational acoustics, J. Comput. Phys. 107 (1993) $262-281$.

[26] C. Bogey, C. Bailly, D. Juvé, Computation of flow noise using source terms in linearized Euler's equations, AIAA J. 40 (2002) $235-243$.

[27] D.H. Johnson, D.E. Dudgeon, Array Signal Processing: Concepts and Techniques (Chapter 4), Prentice Hall, 1993.

[28] T. Brooks, W. Humphreys, A deconvolution approach for the mapping of acoustic sources (DAMAS) determined from phased microphone arrays, J. Sound Vib. 294 (2006) 856-879.

[29] J. Fischer, C. Doolan, Beamforming in a reverberant environment using numerical and experimental steering vector formulations, Mech. Syst. Signal Process. 91 (2017) 10-22. 
[30] S. Candel, Numerical solution of conservation equations arising in linear wave theory: application to aeroacoustics, J. Fluid Mech. 83 (1977) $465-493$.

[31] S. Candel, Sound source radiation in two-dimensional shear flow, AIAA J. 21 (1983) 221-227.

[32] T. Suzuki, D. Bodony, J. Ryu, S.K. Lele, Noise Sources of High-Mach-number Jets at Low Frequencies Studied with a Phased-array Approach Based on LES Database, Annual Research Briefs, Center for Turbulence Research, NASA Ames and Stanford University, 2007.

[33] M. Tanter, J.-L. Thomas, M. Fink, Time reversal and the inverse filter, J. Acoust. Soc. Am. 108 (1) (2000) 223-234. 